\title{
Establishment of a mouse model to express bovine CD14 short hairpin RNA
}

Xiangping $\mathrm{Li}^{1,3^{*}}$, Shihai Huang ${ }^{2}$, Yanping Ren ${ }^{1,3}$, Meng Wang ${ }^{1,3}$, Chao Kang ${ }^{2}$, Liangliang Xie ${ }^{1,3}$ and Deshun Shi ${ }^{1,3^{*}}$

\begin{abstract}
Background: Cluster of differentiation 14 (CD14) functions as a co-receptor for Toll-like receptor (TLR)-4 and myeloid differentiation factor (MD)-2 in detecting bacterial lipopolysaccharide. Together, these complexes promote the phagocytosis and digestion of Gram-negative bacteria, and initiate immune responses. To date, much of our understanding of CD14 function during Gram-negative bacterial inflammation comes from studies on mouse knockout models and cell transfection. To identify the effect of CD14 knockdown in this process in large livestock animals, we established a mouse model expressing bovine CD14 short hairpin (sh) RNA. shRNA fragments targeting bovine CD14 were screened by co-transfection in HEK 293 cells, and the most effective CD14 shRNA fragment was cloned into the eukaryotic expression vector pSilencer4.1-CD14 shRNA-IRES (internal ribosome entry site) and transferred into mouse zygotes by pronuclear microinjection to obtain transgenic mice. Expression of the enhanced green fluorescent protein (EGFP) reporter and genes related to the TLR4 signaling pathway was detected by immunohistochemistry $(\mathrm{IHC})$ and quantitative polymerase chain reaction $(\mathrm{PCR})$, respectively.

Results: One effective shRNA fragment (shRNA-674) targeting bovine CD14 was obtained, the sequence of which was shown to be conserved between cows, buffalos, sheep, and humans. Thirty-seven founder pups were obtained by pronuclear microinjection, of which three were positive for the transgene. In the $F_{1}$ generation, 11 of 33 mice (33\%) were positive for the transgene as detected by PCR. IHC analysis detected exogenous EGFP expression in the liver, kidney, and spleen of transgenic $F_{1}$ mice, indicating that they were chimeric. The expression of endogenous CD14 mRNA in the heart, liver, spleen, lung, and kidney of transgenic $F_{1}$ mice was decreased 8-, 3-, 19.5-, 6-, and 11-fold, respectively. The expression patterns of endogenous MD-2, TLR4, interleukin-6 and tumor necrosis factor- $a$ genes in transgenic mice also varied.
\end{abstract}

Conclusions: This study confirms that transgenic mice expressing bovine CD14 shRNA can be generated by pronuclear microinjection, and demonstrates inhibited endogenous mouse CD14 expression that alters gene expression related to the TLR4 signaling pathway.

Keywords: Bovine CD14, shRNA, Mouse model, TLR4, Gene expression

\section{Background}

$\mathrm{CD} 14$ is a $55 \mathrm{kDa}$ glycoprotein expressed mainly on the surface of monocytes, macrophages, and granulocytes [1], which plays a crucial role in the inflammatory response to lipopolysaccharide (LPS) [2-4]. Currently, LPSinduced cellular activation is thought to occur through signal complexes comprised of CD14 and either myeloid

\footnotetext{
*Correspondence: xiangpingli@163.com; ardsshi@163.com

'State Key Laboratory of Subtropical Bioresource Conservation and Utilization at Guangxi University, Nanning, Guangxi, China

${ }^{3}$ Guangxi High Education Key Laboratory for Animal Reproduction and

Biotechnology, Guangxi University, Nanning 530004, China

Full list of author information is available at the end of the article
}

differentiation factor (MD)-2 or Toll-like receptor (TLR) 4 [5-7]. LPS binding to these complexes facilitates activation of the TLR4/nuclear factor (NF)- $\mathrm{kB}$ inflammatory pathway, ultimately leading to the production of proatherogenic cytokines including tumor necrosis factor alpha (TNF- $\alpha$ ), interleukin-6 (IL-6), and IL-1 [8-11].

Strategies aimed at inhibiting the expression of TLR4 complex genes have been used to analyze their contribution to inflammatory reactions [12-14]. In addition to inhibiting TLR4 expression, many CD14-deficient mice have also been established by CD14 knockout strategies [15-17]. When infected by live Gram-negative bacteria or LPS, CD14-deficient mice demonstrate reduced bacteremia and 
systemic inflammation [18]. Thus, inhibiting signals through CD14 may limit the release of a broad range of inflammatory mediators, and prevent rapid bacterial dissemination following infection by Gram-negative bacteria [1,19-22].

Numerous approaches using monoclonal antibodies, small molecule antagonists, and RNA interference have demonstrated that inhibiting LPS signals through lipopolysaccharide-binding protein, CD14, MD-2, and TLR4 reduce the release of inflammatory cytokines [20,23-26]. For instance, small interfering (si) RNA targeting CD14 in the mouse cell line RAW264.7 was found to inhibit the release of TNF- $\alpha$, macrophage inflammatory protein-2, IL-6, and the production of nitric oxide following exposure to LPS [27]. Thus far, most of our understanding about the role of CD14 during Gram-negative bacterial inflammation comes from studies of mouse knockout models or mouse and human immune cells. However, because of the serious harm caused by bacterial infections such as mastitis and Brucella in large livestock animals and huge resultant losses to the breeding industry, it is essential to establish knockout models of such animals to investigate the CD14 role in LPS-induced inflammation. This would also be of benefit in the development of a practical and effective measure to prevent bacterial infection in livestock. Based on our previous discovery of the effect of $C D 14$ down-regulation in buffalo monocytes/macrophages [28], the present study aimed to establish a transgenic mouse model to express bovine $C D 14$ short hairpin (sh) RNA, and to determine the effect of endogenous mouse CD14 down-regulation on gene expression of the mouse TLR4 signaling pathway.

\section{Results}

\section{Screening of shRNA sequences targeting bovine CD14}

Given the importance of CD14 in LPS signaling, we first sought to screen shRNA sequences for their ability to inhibit bovine CD14 expression in vitro. Using ABI siRNA online software (http://www.ambion.com), three different sites of the bovine CD14 mRNA sequence (GenBank Accession No. NM_174008.1) were used to design three CD14 shRNA sequences (shRNA-279, -326, and -674). CD14 shRNA lentiviral expression vectors with human U6 promoters were constructed (pSicoR-CD14 shRNA279/326/674), and lentiviral particles were produced using the calcium-phosphate method, with titers reaching $1 \times 10^{7}$ (data not shown). Lentiviral particles expressing bovine CD14 shRNA were used to infect HEK 293 cells expressing CD14 at a multiplicity of infection (MOI) of 100, using a non-infected cell line as blank control, the scrambled shRNA as negative control. The infected cells were harvested $72 \mathrm{~h}$ after infection and total RNA was extracted for quantitative reverse-transcription polymerase chain reaction (qRT-PCR) analyses. As expected, cells infected with the shRNA-negative control showed no reduction in CD14 expression (Figure 1A). Compared with scrambled shRNA-1864, shRNA-279 and shRNA326 fragments were also unable to reduce bovine CD14 expression. However, the shRNA-674 fragment significantly inhibited CD14 mRNA expression in vitro $(p<0.01)$ (Figure 1A). shRNA-674 nucleotides were highly conserved between cows, sheep, buffalos, and humans, indicating that this shRNA fragment could potentially be used in related research of multiple species.

To confirm if the shRNA-674 fragment also reduced expression of the CD14 protein, we performed western blot analysis. At two different MOIs, the intracellular expression of shRNA-674 completely abolished CD14 protein expression (Figure 1B), which was consistent with RT-PCR analysis.

\section{shRNA transgenic mice demonstrated reduced CD14 expression}

We next determined the effects of the shRNA-674 fragment on CD14 expression in vivo. To avoid the biological safety problems of lentiviruses, the shRNA-674 fragment was inserted into the eukaryotic shRNA expression vector pSilencer ${ }^{\mathrm{rm}} 4.1-\mathrm{CMV}$ neo. Internal ribosome entry site (IRES) elements were then ligated with the vector to construct the pSilencer4.1-CD14 shRNA-674-IRES plasmid (Figure 1). This plasmid was linearized with Nhe I digestion and microinjected into the pronucleus of fertilized eggs from FVB mice to create CD14 shRNA transgenic mice. After transferring two-cell stage embryos into pseudo-pregnant females, a total of 37 founder pups were obtained. Transgene integration in $F_{0}$ offspring was analyzed by amplifying EGFP and neo genes that were both amplified in three founder mice $(8.1 \%)$ : one male $\left(11^{\#}\right)$ and two females $\left(22^{\#}, 32^{\#}\right)$ that were regarded as transgenic. Within the $F_{1}$ generation (wild-type mouse with transgenic $F_{0}$ mouse cross), 33 mice were born, of which 11 were found to be transgenic (33.3\%; data not shown). In the 11 transgenic F1 mice, 3 was offspring of $11^{\#}$ F0 mouse, 5 and 3 were offspring of $22^{\#}$ and $32^{\#}$ mouse respectively. Three of the $33 \mathrm{~F}_{1}$ mice were selected for Southern blot analysis to confirm the PCR data. Two (lane2, 4, offspring of $22^{\#}, 32^{\#}$ respectively) were transgenic while the third was not (lane3, offspring of $11^{\#}$ ) (Figure 1E). These findings were consistent with PCR results (data not shown).

To further characterize the transgene insertion, organs of $F_{1}$ transgenic mice (offspring of $22^{\#}$ ) were analyzed for eGFP expression by confocal microscopy. This protein was shown to be expressed in the liver, kidney, and spleen tissue of all transgenic mice, with the highest expression detected in the spleen. However, eGFP expression was absent from heart and lung tissues. The expression pattern was similar in both male and female mice (Figure 2). Together these data demonstrated that the transgenic mice were chimeric. 

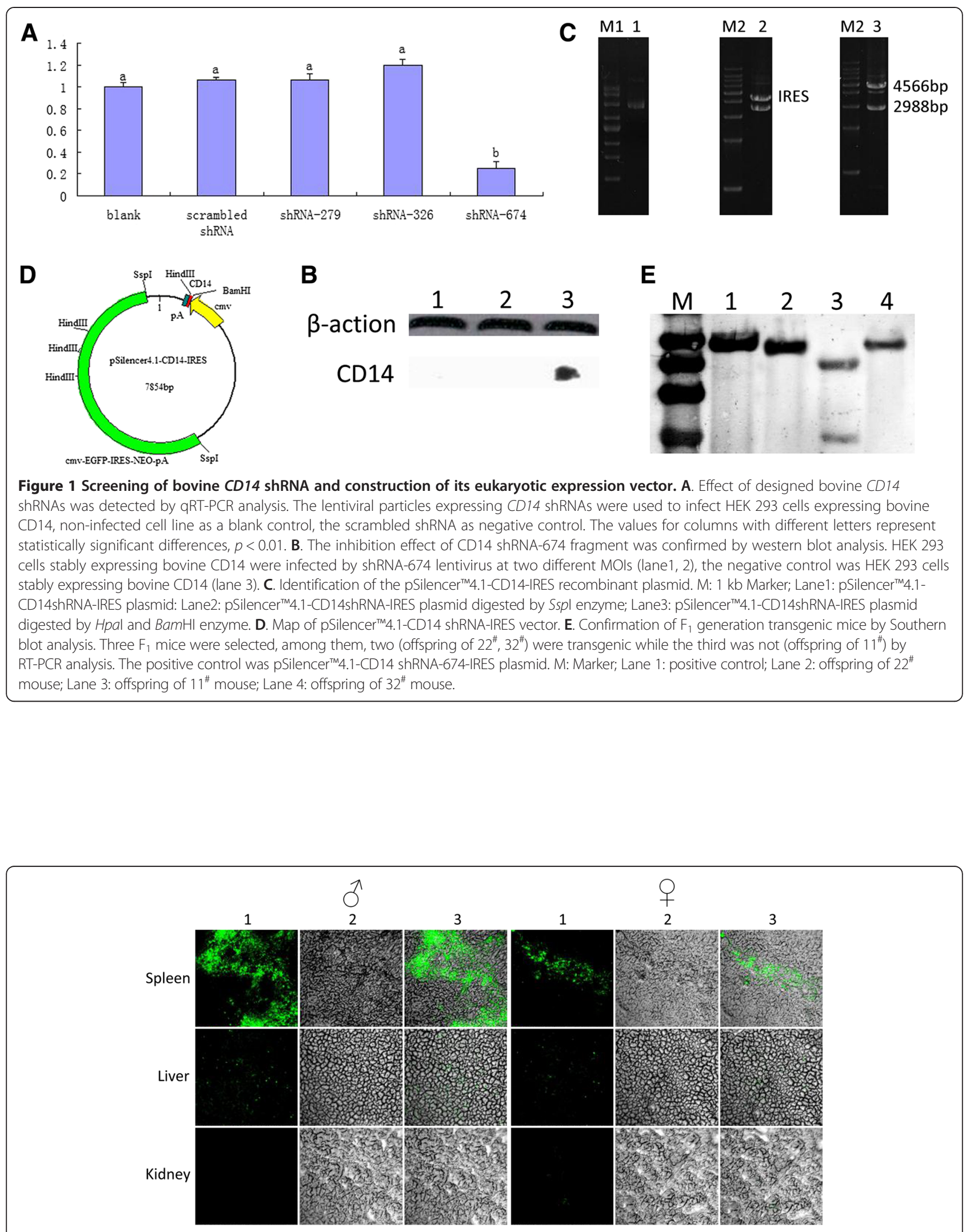

Figure 2 The immunohistochemistry results of eGFP expression in tissues of CD14 shRNA transgenic mice under confocal microscopy. Upper row: spleen; Middle row: liver; Lower row: kidney. Left parts were male samples, and right were female samples. 
To explore if the transferred shRNA fragment affected the expression of endogenous CD14 mRNA in vivo, we used qRT-PCR to analyze the relative expression of CD14 expression in tissues of $\mathrm{F}_{1}$ transgenic mice (offspring of $\left.22^{\#}\right)$. Compared with non-transgenic mouse RNA samples, the expression of endogenous CD14 mRNA in the heart, liver, spleen, lung, and kidney tissues of transgenic mice was reduced 8-, 3-, 19.5-, 6-, and 11 fold, respectively (Figure 3). Thus, the expression of endogenous CD14 mRNA was inhibited in transgenic mice.

We next examined the expression pattern of genes in the TLR4 signaling pathway. TLR4 demonstrated a significantly increased expression level in the heart and liver of CD14 shRNA transgenic mice compared with wild-type mice $(p<0.05)$, although there were no significant differences in expression in the spleen, lung, or kidney between transgenic and wild-type mice. $M D-2, T N F-\alpha$, and $I L-6$ transcripts showed similar expression patterns, except for the kidney, with significantly increased expression in the heart, liver, and lung $(p<0.05)$, and significantly lower expression in the spleen $(p<0.05)$ compared with wild-type mice. Exogenous neo expression showed the same pattern as that of $M D-2$ and $I L-6$ (Figure 3) but with greater differences between transgenic and wild-type mice. Together, these results revealed the successful generation of a mouse model expressing bovine CD14 shRNA, and indicated that the inhibition of exogenous CD14 expression altered the expression levels of genes in the TLR4 signaling pathway in vivo.

\section{Discussion}

Several studies have previously demonstrated that CD14 plays a crucial role in the inflammatory response to LPS [2-4]. In the CD14-dependent signaling pathway, CD14 binds to LPS and facilitates activation of the TLR4/NF- $\mathrm{kB}$ inflammatory pathway [28]. Upstream inhibition of the bacterial LPS/TLR4/CD14-mediated inflammation pathway has been proven to be an effective therapeutic approach for attenuating dysfunctional immune activation $[20,29]$. However, very few studies have investigated the role of CD14 in LPS-induced inflammation in large livestock animals such as sheep, cows, and buffalos. Nevertheless, this is of particular importance because of the multiple reproductive and veterinary problems associated with these species, and the need to develop a practical and effective measure to prevent bacterial infections in livestock.

We previously found that knockdown of endogenous CD14 had clear regulatory effects on the signal transduction of TLR4 after stimulation with LPS in buffalo monocyte/macrophages in vitro [28]. To determine if CD14 knockdown had similar effects in large livestock animals in vivo, it is first necessary to establish a mouse

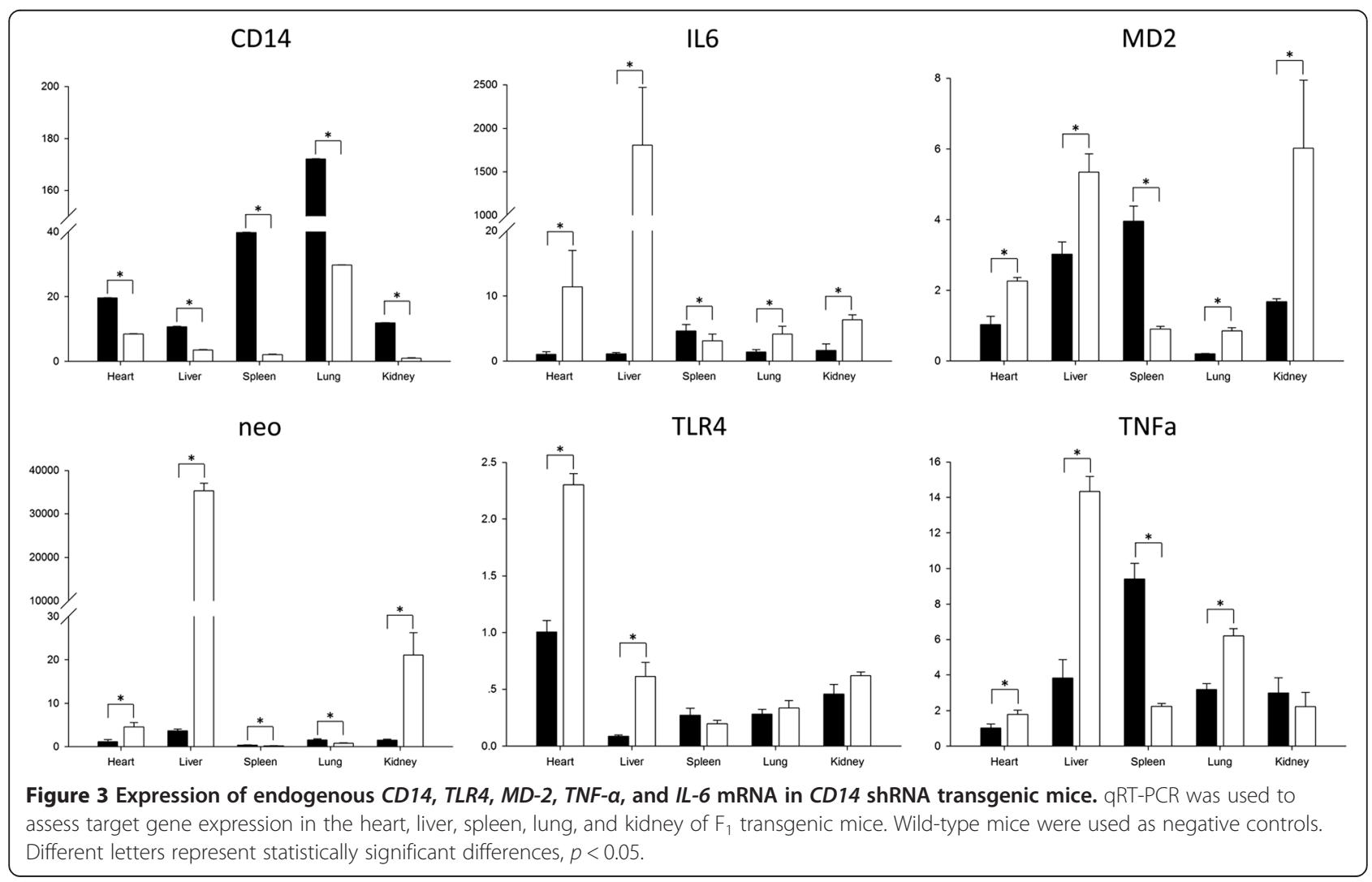


model expressing bovine CD14 shRNA. This model would provide basic data about CD14 knockdown on animal development, gene expression in the CD14dependent signaling pathway, and most importantly on toxicity experiments. Future work could then build on these data and establish a larger animal model with the aim of developing novel therapeutic interventions to inflammatory diseases caused by Gram-negative bacteria.

\section{Conclusions}

We successfully generated a mouse model expressing bovine $C D 14$ shRNA by pronuclear microinjection. Moreover, we showed that the inhibited expression of exogenous CD14 shRNA altered the expression levels of some genes in the TLR4 signaling pathway in transgenic mice.

\section{Methods}

All experiments and protocols were performed in strict accordance with the Guiding Principles for the Care and Use of Research Animals from the Guangxi University Committee on Animal Research and Bioethics, the committee explicitly approved the animal study.

\section{Reagents and antibodies}

All chemicals used in this study were purchased from Sigma-Aldrich (St. Louis, MO), unless otherwise stated. TCM-199 powder was purchased from Gibco BRL (Paisley, Scotland, UK), and Dulbecco's modified Eagle's medium was purchased from Hyclone (Logan, UT). The pSilencer4.1 vector (Life technology, USA), the pEF-EGFP-IRESneo-SV40-polyA vector, and the pSicoR-GFP vector were generated or maintained by our laboratory. The antibuffalo CD14 primary antibody was kindly provided by Dr. Wang Fengyang (Hainan University, Haikou, China).

\section{Bovine CD14 cloning and expression vector construction} The bovine CD14 coding sequence fragment (1,340 bp) was cloned by RT-PCR, confirmed by sequencing, and used to construct the pDsRed1-N1-bovine CD14 fusion vector by inserting the CD14 fragment into the Salland
SacII sites. This plasmid was then transfected into HEK 293 cells using Lipofectamine ${ }^{\bullet}$ LTX reagent according to the manufacturer's instructions, and cell lines that stably expressed bovine CD14 were selected using G418 selection.

\section{shRNA design and synthesis}

Using ABI siRNA online software (http://www.life technologies.com/cn/zh/home/life-science/rnai/syntheticrnai-analysis/ambion-silencer-select-sirnas/silencer-selectsirna.html?ICID=search-am16704), three different regions of the bovine CD14 mRNA sequence (GenBank Accession No. NM_174008.1) were used to design CD14 shRNA sequences, which were synthesized by Nanjing GenScript Co. (Nanjing, China). A universal shRNA scramble control (NC) sequence was also purchased (Cat. No. 1864, Open Biosystem, Huntsville, AL). The 71 bp oligonucleotide sequence of each shRNA fragment followed the same pattern: 5'-Xho I-CCGG-shRNA (sense strand)-TTGAA GAGA (loop structure)-shRNA (antisense strand)-TTTT TT-Not I-3' (Table 1). shRNA lentiviral expression vectors were constructed by inserting the synthesized shRNA fragments into the pSicoR-GFP vector (Addgene, USA) after digestion with $X h o$ I and Not I. These vectors are referred to as pSicoR-GFP-CD14 shRNA (279/326/674) and scrambled shRNA-1864. The constructed shRNA lentiviral vectors were confirmed by restriction enzyme digestion and sequencing.

\section{Lentivirus packaging and titer determination}

Lentiviral particles were produced as previously described [30]. The pSicoR-GFP CD14 shRNA vector was co-transfected into $293 \mathrm{~T}$ cells with vesicularstomatitis virusG (VSVG) and NRF plasmids using the calciumphosphate method [30]. Supernatant was harvested 48-72 $\mathrm{h}$ after transfection, centrifuged at 2,000 rpm for $10 \mathrm{~min}$ at $4^{\circ} \mathrm{C}$ to remove cellular debris, and filtered through a $0.45 \mu \mathrm{m}$ membrane. Viral titers were determined using a serial dilution method in 293 T cells [30].

Table 1 The designed shRNA sequences of the bovine CD14 gene

\begin{tabular}{|c|c|}
\hline Name & Duplexes of DNA coding specific shRNA $\left(5^{\prime}-3^{\prime}\right)$ \\
\hline \multirow[t]{2}{*}{ shRNA-674 } & S 5 '-GCCTAGACCTGTCTGACAATTTCAAGAGAATTGTCAGACAGGTCTAGGC-3' \\
\hline & AS 5'-CGGATCTGGACAGACTGTTAAAGTTCTCTTAACAGTCTGTCCAGATCCG-3' \\
\hline \multirow[t]{2}{*}{ shRNA-279 } & S 5'-GCCTGGAACAGTTTCTCAAGGTTCAAGAGACCTTGAGAAACTGTTCCAGGC-3' \\
\hline & AS 5'-CGGACCTTGTCAAAGAGTTCCAAGTTCTCTGGAACTCTTTGACAAGGTCCG-3' \\
\hline \multirow[t]{2}{*}{ shRNA-326 } & S 5'-GCTGACACAATCAAGGCTCTGTTCAAGAGACAGAGCCTTGATTGTGTCAGC-3' \\
\hline & AS 5'-CGACTGTGTTAGTTCCGAGACAAGTTCTCTGTCTGGCAACTAACACAGTCG-3' \\
\hline \multirow[t]{2}{*}{ Scrambled shRNA-1864 } & S 5'-CTCGAGCCGGCCTAAGGTTAAGTCGCCCTCGCTCG AGCGAGGGCGACTTAACCTTAGGTIIITGGCGGCCGC-3' \\
\hline & AS 5'-GCGGCCGCCAAAAAACCTAAGGTTAAGTCGCCCTC GCTCGAGCGAGGGCGACTTAACCTTAGGCCGGCTCGAG-3' \\
\hline
\end{tabular}


Screening of shRNA sequences targeting bovine CD14 shRNA sequences were screened by infecting HEK293 cells expressing bovine CD14 with lentiviral particles containing different $C D 14$ shRNAs. Cells were harvested $72 \mathrm{~h}$ after infection and total RNA was extracted using Trizol reagent. The inhibition effects of each shRNA sequence on CD14 were quantified using qRT-PCR and western blot analysis.

For qRT-PCR analysis, total RNA was extracted with Trizol (Invitrogen, USA), digested with DNaseI (Tiangen, Beijing) to remove contaminating genomic DNA, then reverse transcribed into cDNA using AMV reverse transcriptase (Takara, Dalian) according to the manufacturer's instructions. cDNA was diluted to $100 \mathrm{ng} / \mu \mathrm{L}$ for subsequent TaqMan quantitative PCR analysis (ABI 7500) using the probes and primers listed in Table 2. PCR conditions were: $94^{\circ} \mathrm{C}$ for $30 \mathrm{~s}$, followed by 40 cycles of $94^{\circ} \mathrm{C}$ for $15 \mathrm{~s}$ and $60^{\circ} \mathrm{C}$ for $30 \mathrm{~s}$. Duplicate PCR experiments were performed for each transcript. The comparative $\mathrm{Ct}$ method was used for the relative quantification of target gene expression levels (ABI Prism Sequence Detection System). The histone H2a gene was used for normalization. Within the log-linear phase region of the amplification curve, fold-changes in the relative mRNA expression of the target gene were determined using the formula $2^{-\Delta \Delta C T}$.
Western blot analysis was performed using standard protocols. The primary antibody was a rabbit anti-bovine CD14 polyclonal (1:200) (a gift from Dr. Fengyang Wang), and the secondary antibody was horseradish peroxidaseconjugated goat anti-rabbit IgG (Tiangen Biotech, Beijing, China; 1:1,000). Bovine CD14 shRNA-674 lentivirus was used to infect HEK 293 cells stably expressing bovine CD14 at two different MOIs, 50 and 100 respectively. The cells were harvested at $72 \mathrm{~h}$ after infection, HEK 293 cells stably expressing bovine CD14 were used as negative controls.

\section{Construction of CD14 shRNA-674 eukaryotic expression vector}

The pSilencer ${ }^{\mathrm{rm}} 4.1-\mathrm{CMV}$ neo and pSicoR-CD14 shRNA674 plasmids were first digested with $\mathrm{BamHI}$ and HindIII, respectively, then the CD14 shRNA-674 fragment was inserted into the pSilencer ${ }^{\text {im }} 4.1-\mathrm{CMV}$ neo backbone to construct the pSilencer ${ }^{\text {rm }} 4.1-C D 14$ shRNA plasmid. The IRES fragment was amplified by PCR using pEFEGFP-IRES-neo-SV40-polyA vector as template and primer sequences listed in Table 2. It was then ligated with pSilencer ${ }^{\text {rw }} 4.1-C D 14$ shRNA-674 to construct the pSilencer ${ }^{\mathrm{mex}} 4.1$-CD14 shRNA-674-IRES plasmid. Plasmid

Table 2 The primers used in the paper

\begin{tabular}{|c|c|c|}
\hline Gene & Primer sequences & Fragment length (bp) \\
\hline \multirow[t]{3}{*}{ Histone $\mathrm{H} 2 \mathrm{a}$} & Forward: 5'-AACAAGCTGCTGGGCAAAGT-3' & 80 \\
\hline & Reverse: 5'-TTATGGTGGCTCTCCGTCTTCT-3' & \\
\hline & Probe: 5'-CCCAACATCCAGGCCGTGCTG-3' & \\
\hline \multirow[t]{3}{*}{ CD14 } & Forward: 5'-CCGTTCAGTGGTAATGGTTGC-3' & 100 \\
\hline & Reverse: 5'-TGGTGTCGGCTCCCTTGAG-3' & \\
\hline & Probe: 5'-CCGCCCGCCACTGATCTTCCCACCTCTT-3' & \\
\hline \multirow[t]{2}{*}{ EGFP } & Forward: 5'- ACGTAAACGGCCACAAGTTC -3' & 440 \\
\hline & Reverse: 5'- GATCTTGAAGTTCACCTTGATGC -3' & \\
\hline \multirow[t]{2}{*}{$\mathrm{Neo}$} & Forward: 5'- AGAGGCTATTCGGCTATGAC -3' & 211 \\
\hline & Reverse: 5'-GCTTCAGTGACAACGTCGAG -3' & \\
\hline \multirow[t]{2}{*}{ IRES } & Forward: 5'-CGGAATATTATAACTTCGTATAATGTATGCTATACGAAGTTATCTTCCGACATTGATTATTGAC-3' & 4300 \\
\hline & Reverse: 5'-CGGAATATTATAACTTCGTATAGCATACATTATACGAAGTTATGATCCAGACATGATAAGATAC-3' & \\
\hline \multirow[t]{2}{*}{ TLR4 } & Forward: 5'-CTGCCTGAGAACCGAGAGTTG-3' & 300 \\
\hline & Reverse: 5'-GCTCCATGCACTGGTAACTAATGT-3' & \\
\hline \multirow[t]{2}{*}{ IL-6 } & Forward: 5'- ATCAGAACACTGATCCAGATCC-3' & 300 \\
\hline & Reverse: 5'-CAAGGTTTCTCAGGATGAGG-3' & \\
\hline \multirow[t]{2}{*}{ TNF-a } & Forward: 5'-GCTCCAGAAGTTGCTTGTGC-3' & 300 \\
\hline & Reverse: 5'-AACCAGAGGGCTGTTGATGG-3' & \\
\hline \multirow[t]{2}{*}{ MD2 } & Forward: GAGTTGCCGAAGCGTAAG & 213 \\
\hline & Reverse: GCGGTGAATGATGGTGAA & \\
\hline \multirow[t]{2}{*}{$\beta$-actin } & Forward: 5'-GCCCTGGCACCCAGCACAAT-3' & 150 \\
\hline & Reverse: 5'-GGAGGGGCCGGACTCATCGT-3' & \\
\hline
\end{tabular}


construction was confirmed by enzyme digestion and cell transfection experiments.

\section{Generation and detection of transgenic mice}

The pSilencer ${ }^{\mathrm{ma}} 4.1$-CD14 shRNA-674-IRES cassette was digested with Nhe I (Takara, Dalian, China) and purified by gel extraction (Qiagen, USA). The purified fragment was then microinjected into the pronucleus of 200 fertilized FVB mice eggs, which were implanted into pseudopregnant FVB females. All mice were housed at the transgenic mouse facility of Cyagen Biosciences Inc. (Guangzhou, China).

DNA purified from the tails of $F_{0}$ offspring mice was used to screen for transgene integration by the PCR amplification of EGFP and neo (Table 2). Transgenic $\mathrm{F}_{0}$ mice were crossed with wild-type mice to obtain an $F_{1}$ generation that was also screened using PCR amplification as above. Three of the $33 F_{1}$ mice were selected for Southern blot analysis to confirm PCR data using the DIG High Prime DNA Labeling and Detection Starter kit II (Roche, USA). The probe was complemented with the neo fragment by digesting the pSilencer4.1-CD14shRNA-IRES plasmid with Nco I and Eag I. The positive control was pSilencer ${ }^{\mathrm{rm}} 4.1-C D 14$ shRNA-674-IRES plasmid.

\section{Transgene expression analysis}

The expression of eGFP in tissues of $F_{1}$ transgenic mice was assayed by confocal microscopy (LSM 510, Zeiss, Oberkochen, Germany). Briefly, 10- $\mu$ m-thick cryostat sections from snap frozen tissues were prepared and fixed with $4 \%$ paraformaldehyde. Specimens were visualized using an Olympus BX61 microscope, and digital pictures were acquired with a CCD camera.

The relative expression of CD14, MD-2, TLR4, IL-6, $T N F-\alpha$, and neo mRNA in the heart, liver, spleen, lung, and kidney of $F_{1}$ transgenic mice was assessed by SYBR $^{\circ}$ Green qRT-PCR; tissues of wild-type mice were used as a negative control. ACTB and histone $\mathrm{H} 2 \mathrm{a}$ were used as internal control genes (Table 2), and non-transcribed RNA samples served as an RT-minus control. The expression level of each target gene was calculated using the $2^{-\Delta \Delta C T}$ formula as above.

\section{Statistical analysis}

qRT-PCR mRNA expression data were analyzed using SPSS16.0 and Excel 2003 to statistically process multiple samples. Single-factor analysis of variance and the q-test were used for pairwise comparisons. A $P$-value of less than 0.05 was considered to be significant.

\section{Abbreviations}

CD14: Cluster of differentiation antigen 14; LPS: Lipopolysaccaride; TLR: Toll-like receptor; IL-6: Interleukin-6; TNF-a: Tumor necrosis factor-a; NF-KB: Nuclear factor kappaB; shRNA: Short hair RNA; MOI: Multiplicity of infection.
Competing interests

The authors declare that they have no competing interests.

\section{Authors' contributions}

XPL drafted the manuscript and participated in all study design. SHH participated in gene expression assays and virus package. YPR constructed the CD14 shRNA-674 eukaryotic expression vector and participated in some gene expression assays. MW analyzed the trangenic mouse. CK cloned the bovine CD14 gene and screened the shRNAs. LLX participated in mouse breeding. DSS conceived the study and helped draft the manuscript. All authors read and approved the final manuscript.

\section{Acknowledgements}

This work was supported by the National Transgenic Project (2009ZX08007-009B), Guangxi Natural Science funding (2012GXNSFCB053002), funding from the State Key Laboratory of Subtropical Bioresource Conservation and Utilization (KSL-CUSAb-2012-02) and State Education Ministry's Scientific Research Foundation for the Returned Overseas Chinese Scholars.

\section{Author details}

${ }^{1}$ State Key Laboratory of Subtropical Bioresource Conservation and Utilization at Guangxi University, Nanning, Guangxi, China. ${ }^{2}$ College of Life Science and Technology, Guangxi University, Nanning, Guangxi, China. ${ }^{3}$ Guangxi High Education Key Laboratory for Animal Reproduction and Biotechnology, Guangxi University, Nanning 530004, China.

Received: 10 June 2014 Accepted: 6 February 2015

Published online: 15 February 2015

\section{References}

1. Ziegler-Heitbrock HW, Ulevitch RJ. CD14: cell surface receptor and differentiation marker. Immunol Today. 1993;14:121-5.

2. Le Roy D, Di Padova F, Adachi Y, Glauser MP, Calandra T, Heumann D. Critical role of lipopolysaccharide-binding protein and CD14 in immune responses against gram-negative bacteria. J Immunol. 2001;167:2759-65.

3. Song G, Li X, Shen Y, Qian L, Kong X, Chen M, et al. Transplantation of iPSC Restores Cardiac Function by Promoting Angiogenesis and Ameliorating Cardiac Remodeling in a Post-infarcted Swine Model. Cell Biochem Biophys. 2014; [Epub ahead of print].

4. Li XQ, Pryds A, Carlsen J, Larsen M. Multifocal central serous chorioretinopathy with photoreceptor-retinal pigment epithelium diastasis in heritable pulmonary arterial hypertension. Retinal Cases Brief Rep. 2015:9:83-7

5. Thorgersen EB, Hellerud BC, Nielsen EW, Barratt-Due A, Fure H, Lindstad JK, et al. CD14 inhibition efficiently attenuates early inflammatory and hemostatic responses in Escherichia coli sepsis in pigs. FASEB J. 2010;24:712-22.

6. Li X, Cai J, Zhuang Z, Liu J, Xia B, Hu G, et al. Investigation of the action mechanisms of poly-ADP-ribosylation in hexavalent chromium induced cell damage. Zhonghua Yu Fang Yi Xue Za Zhi. 2014;48:720-5.

7. Zuo H, Liao D, Lin L, Zhang R, Li X. Resveratrol attenuates hypoxiareperfusion injury induced rat myocardium microvascular endothelial cell dysfunction through upregulating PI3K/Akt/SW pathways]. Zhonghua Xin Xue Guan Bing Za Zhi. 2014:42:670-4.

8. Miyake K. Innate immune sensing of pathogens and danger signals by cell surface Toll-like receptors. Semin Immunol. 2007;19:3-10.

9. Miyake K. Innate recognition of lipopolysaccharide by CD14 and toll-like receptor 4-MD-2: unique roles for MD-2. Int Immunopharmacol. 2003;3:119-28.

10. Miyake K. Innate recognition of lipopolysaccharide by Toll-like receptor 4-MD-2. Trends Microbiol. 2004;12:186-92.

11. Takeuchi O, Akira S. Pattern recognition receptors and inflammation. Cell. 2010;140:805-20.

12. Chen S, Lee LF, Fisher TS, Jessen B, Elliott MW, Evering W, et al. Combination of 4-1BB agonist and PD-1 antagonist promotes anti-tumor effector/memory CD8 T cells in a poorly immunogenic tumor model. Cancer Immunol Res. 2015;3:149-60.

13. Xu Z, Huang CX, Li Y, Wang PZ, Ren GL, Chen CS, et al. Toll-like receptor 4 siRNA attenuates LPS-induced secretion of inflammatory cytokines and chemokines by macrophages. J Infect. 2007:55:e1-9. 
14. Mancek-Keber M, Gradisar H, Pestana MI, de Tejada GM, Jerala R. Free Thiol Group of MD-2 as the Target for Inhibition of the Lipopolysaccharideinduced Cell Activation. J Biol Chem. 2009;284:19493-500.

15. Qian L, Zhao H, Li X, Yin J, Tang W, Chen P, et al. Pirenzepine Inhibits Myopia in Guinea Pig Model by Regulating the Balance of MMP-2 and TIMP-2 Expression and Increased Tyrosine Hydroxylase Levels. Cell Biochem Biophys 2014. [Epub ahead of print].

16. Haziot A, Ferrero E, Kontgen F, Hijiya N, Yamamoto S, Silver J, et al. Resistance to endotoxin shock and reduced dissemination of gram-negative bacteria in CD14-deficient mice. Immunity. 1996;4:407-14.

17. Roncon-Albuquerque R, Moreira-Rodrigues M, Faria B, Ferreira AP, Cerqueira C, Lourenco AP, et al. Attenuation of the cardiovascular and metabolic complications of obesity in CD14 knockout mice. Life Sci. 2008;83:502-10.

18. Haziot A, Rong GW, Lin XY, Silver J, Goyert SM. Recombinant soluble CD14 prevents mortality in mice treated with endotoxin (lipopolysaccharide). J Immunol. 1995;154:6529-32.

19. Li XX, Guan HJ, Liu JP, Guo YP, Yang Y, Niu YY, et al. Association of selenoprotein $\mathrm{S}$ gene polymorphism with ischemic stroke in a Chinese case-control study. Blood Coagul Fibrinolysis. 2015;26:131-5.

20. Nagaoka I, Hirota S, Niyonsaba F, Hirata M, Adachi Y, Tamura H, et al. Cathelicidin family of antibacterial peptides CAP18 and CAP11 inhibit the expression of TNF-alpha by blocking the binding of LPS to CD14 (+) cells. J Immunol. 2001;167:3329-38.

21. Li X, Lu C, Dai J, Dong S, Chen Y, Hu N, et al. Novel multiferroicity in GdMnO3 thin films with self-assembled nano-twinned domains. Sci Rep. 2014;4:7019. doi:10.1038/srep07019.

22. Hou Y, Wang Y, Zhao J, Li X, Cui J, Ding J, et al. Smart soup, a traditional chinese medicine formula, ameliorates amyloid pathology and related cognitive deficits. PLoS One. 2014;9:e111215.

23. Mookherjee N, Wilson HL, Doria S, Popowych Y, Falsafi R, Yu JJ, et al. Bovine and human cathelicidin cationic host defense peptides similarly suppress transcriptional responses to bacterial lipopolysaccharide. J Leukocyte Biol. 2006;80:1563-74.

24. Nemchinov LG, Paape MJ, Sohn EJ, Bannerman DD, Zarlenga DS, Hammond RW. Bovine CD14 receptor produced in plants reduces severity of intramammary bacterial infection. Faseb J. 2006;20:1345-51.

25. Song YX, Dou H, Gong W, Liu XQ, Yu ZG, Li EG, et al. Bis-N-norgliovictin, a small-molecule compound from marine fungus, inhibits LPS-induced inflammation in macrophages and improves survival in sepsis. Eur J Pharmacol. 2013;705:49-60.

26. Shao Y, Cheng Z, Li X, Chernaya V, Wang H, Yang XF. Immunosuppressive/ anti-inflammatory cytokines directly and indirectly inhibit endothelial dysfunction- a novel mechanism for maintaining vascular function. J Hematol Oncol. 2014;7:80.

27. Li X, Kolomeisky AB. Theoretical analysis of microtubule dynamics at all times. J Phys Chem B. 2014;118:13777-84.

28. Li X, Li M, Huang S, Qiao S, Qin Z, Kang C, et al. The effect of buffalo CD14 shRNA on the gene expression of TLR4 signal pathway in buffalo monocyte/macrophages. Cell Mol Biol Lett. 2014;19:623-37.

29. Verbon A, Dekkers PE, ten Hove T, Hack CE, Pribble JP, Turner T, et al. IC14, an anti-CD14 antibody, inhibits endotoxin-mediated symptoms and inflammatory responses in humans. J Immunol. 2001;166:3599-605.

30. Li X, Hou L, Liu M, Lin X, Li Y, Li S: Primary effects of extracellular enzyme activity and microbial community on carbon and nitrogen mineralization in estuarine and tidal wetlands. Applied microbiology and biotechnology 2014. [Epub ahead of print].

\section{Submit your next manuscript to BioMed Central and take full advantage of:}

- Convenient online submission

- Thorough peer review

- No space constraints or color figure charges

- Immediate publication on acceptance

- Inclusion in PubMed, CAS, Scopus and Google Scholar

- Research which is freely available for redistribution 